\title{
SOME REMARKS CONCERNING THE VARIETIES GENERATED BY THE DIAMOND AND THE PENTAGON $\left({ }^{1}\right)$
}

BY

\author{
S. D. COMER AND D. X. HONG
}

ABSTRACT. In 1945 M. P. Schützenberger exhibited two identities. He asserted that one provided an equational base for the diamond $M_{3}$ and the other a base for the pentagon $N_{5}$. Recently Ralph McKenzie produced another equational base for $N_{5}$. In the present paper the authors modify McKenzie's idea to verify Schützenberger's assertion for $M_{3}$. They also show Schützenberger's claim about $N_{5}$ is false.

Introduction. In this note we make some observations based on the preceding paper [2] by R. McKenzie. In $\$ 1$ we modify the ideas in $\$ 2$ of McKenzie's paper to obtain analogous results for $\mathcal{M}_{3}$, the variety of lattices generated by the diamond. In particular, we provide a proof of the result announced by Schützenberger [3] that $O_{M_{3}}$ is characterized by the single identity:

a.

$$
\begin{aligned}
x \cdot(y+z \cdot(u+v))= & x \cdot(y+z u)+x \cdot(y+z v)+x z \cdot(u+v) \\
& +x u \cdot(z+y v)+x v \cdot(z+y u) .
\end{aligned}
$$

This fact also follows from the much stronger results of Jónsson [1]; however, our proof of this result, like McKenzie's proof that certain identities characterize $\mathrm{ON}_{5}$, is model-theoretic in nature while Jónsson's results involve deeper lattice theoretic techniques.

In the article cited above, Schützenberger also asserted without proof that the variety $\mathcal{O}_{5}$ generated by the pentagon is characterized by the identity: $\beta$.

$$
x \cdot[y+z \cdot(u+v)]=x \cdot(x y+z u)+x \cdot(y+x z u)+x \cdot(x y+z v)
$$

$$
+x \cdot(y+x z v)+x z \cdot(x z u+v)+x z \cdot(u+x z v) .
$$

In $\S 2$ of our note we observe that $\beta$ holds in some lattice not contained in $\theta_{N_{5}}$; thus $\beta$ does not characterize $\mathcal{O} N_{5}$. Equational bases for $\mathcal{O} N_{5}$ have been found by McKenzie (see [2]).

The authors wish to thank Professors Jónsson and McKenzie for their several

Received by the editors March 5, 1969 and, in revised form, May 6, 1971.

AMS (MOS) subject classifications (1970). Primary 06A30, 08A15; Secondary 06A20, 06A35.

Key words and phrases. Variety of lattices, equational base, Schützenberger identities, modular law, distributive law.

$\left({ }^{1}\right)$ Research supported by NSF Grants GP 7252 and GP 8725. 
stimulating conversations concerning the questions considered here and in particular Professor McKenzie for making available an early version of his results.

1. Following McKenzie, a special term of type one (an ST1) is any lattice term of the form $\rho \cdot(\sigma+\tau)$ where $\rho, \sigma, \tau$ are each products of variables. A term in the dual form is called an ST2. McKenzie proved that for any ST1 $\nu$ and ST2 $\phi$ the inclusion $\nu \leq \phi$ (called a special inclusion) either holds in every lattice or else implies (modulo lattice theory $\Lambda$ ) the modular law. We will modify McKenzie's proof to show the following.

Lemma 1.1. Every special inclusion $\epsilon$ either bolds in all modular lattices or else implies (modulo $\Lambda$ ) the distributive law.

If $\Theta$ is any equational theory we write $\sigma \leq_{\boldsymbol{\theta}} \tau$ instead of $\sigma \leq \tau \in \Theta$ and $\sigma \sim_{\theta} \tau$ in place of $\sigma=\tau \in \Theta$. The equational theory of modular lattices is denoted by $\mathrm{M}$; distributive lattices by $\Delta$. The following is an analogue of McKenzie's Lemma 2.2.

Lemma 1.2. For each term $\sigma$, there are finite, nonempty sets of terms $F_{1}$ and $F_{2}$ such that

(i) $F_{1}$ consist of ST1's $\nu$ satisfying $\nu \leq_{M} \sigma$; moreover $\sigma=\Sigma F_{1} \in \boldsymbol{\Theta}_{l}[a]$;

(ii) $F_{2}$ consist of ST2's $\phi$ satisfying $\sigma \leq_{M} \phi$; moreover $\sigma=\Pi F_{2} \in \mathbf{\Theta}_{l}[a]$.

As an application of Lemmas 1.1 and 1.2 we prove $\alpha$ characterizes $0 M_{3}$.

Theorem 1.3. $\boldsymbol{\Theta} M_{3}=\boldsymbol{\Theta}_{l}[a]$.

As an elementary application of the model-theoretic ideas to be used in the proof of 1.3 we first give a simple proof of the following well-known theorem.

Theorem 1.4. The variety of lattices generated by the two element chain is the class of all lattices satisfying the distributive law $x \cdot(y+z)=x y+x z$.

Proof. Clearly the two element chain satisfies the distributive law. Since the distributive law is self-dual, it is easily seen that from this law and $\Lambda$ every term is equivalent to a term $\Sigma \sigma_{i}$ where $\sigma_{i}$ is a product of variables and also to a term $\Pi \tau_{j}$ where $\tau_{j}$ is a sum of variables. Thus, every lattice inclusion $\nu \leq \phi$ is equivalent to a conjunction of inclusions of the form $\sigma \leq \tau$ where $\sigma$ is a product and $\tau$ a sum of variables. Now, an inclusion $\sigma \leq \tau$ of this form will either hold in every lattice or fail in the two element chain depending upon whether or not some variable occuring in $\sigma$ also occurs in $\tau$. Thus, every identity holding in the two element chain follows from the distributive law and $\Lambda$.

In view of McKenzie's Lemma 2.1, our Lemma 1.1 and the above proof every special inclusion is either a lattice identity or equivalent to the modular law, distributive law or $x=y$. This fact was also observed independently by McKenzie. 
Proof of Theorem 1.3. From Lemma 1.2 it follows that modulo $\boldsymbol{\Theta}_{l}[\alpha]$ every equation satisfied by the diamond $M_{3}$ is equivalent to a conjunction of special inclusions. Such a special inclusion does not imply the distributive law; thus, by Lemma 1.1 it belongs to $M$. It is easily seen (and we will prove later) that $\alpha$ implies the modular law; thus, $\Theta_{3} \subseteq \boldsymbol{\Theta}_{l}[a]$.

It remains to show that $a$ holds in $M_{3}$ which we do directly. Suppose the variables $x, y, z, u$, and $v$ are, respectively, assigned to elements $x^{\prime}, y^{\prime}, z^{\prime}, u^{\prime}$ and $v^{\prime}$ in $M_{3}$. The right (resp. left) side of $\alpha$ is then assigned to an element RS (resp. LS) in $M_{3}$. By modularity each summand on the right side of $\alpha$ is contained in LS; hence it suffices to show LS is always a sum of elements on the right.

Whenever $u^{\prime}$ and $v^{\prime}$ are comparable or one of $x^{\prime}, y^{\prime}, z^{\prime} \in\{0,1\}$, LS $\leq \mathrm{RS}$ is easily checked. Now assume $u^{\prime}$ and $v^{\prime}$ are incomparable and $x^{\prime}, y^{\prime}, z^{\prime} \notin\{0,1\}$. Since $u^{\prime}+v^{\prime}=1$, it suffices to show

$$
\begin{aligned}
x^{\prime} \cdot\left(y^{\prime}+z^{\prime}\right)= & x^{\prime} \cdot\left(y^{\prime}+z^{\prime} u^{\prime}\right)+x^{\prime} \cdot\left(y^{\prime}+z^{\prime} v^{\prime}\right) \\
& +x^{\prime} z^{\prime}+x^{\prime} u^{\prime}\left(z^{\prime}+y^{\prime} v^{\prime}\right)+x^{\prime} v^{\prime} \cdot\left(z^{\prime}+y^{\prime} u^{\prime}\right) .
\end{aligned}
$$

Moreover, we may assume that $x^{\prime}, y^{\prime}, z^{\prime}$ are mutually incomparable; for if $y^{\prime}, z^{\prime}$ are comparable then $x^{\prime} \cdot\left(y^{\prime}+z^{\prime}\right)=x^{\prime} \cdot\left(y^{\prime}+z^{\prime} u^{\prime}\right)+x^{\prime} z^{\prime}$ and, if $x^{\prime}$ is comparable with $y^{\prime}$ or $z^{\prime}$, then

$$
x^{\prime} \cdot\left(y^{\prime}+z^{\prime}\right)=x^{\prime} y^{\prime}+x^{\prime} z^{\prime}=x^{\prime} \cdot\left(y^{\prime}+z^{\prime} u^{\prime}\right)+x^{\prime} z^{\prime}
$$

by modularity. Thus, there are only three remaining cases: $z^{\prime}=u^{\prime}, z^{\prime}=v^{\prime}$, $\left\{x^{\prime}, y^{\prime}\right\}=\left\{u^{\prime}, v^{\prime}\right\}$. In the first two cases $x^{\prime} \cdot\left(y^{\prime}+z^{\prime}\right)=x^{\prime} \cdot\left(y^{\prime}+z^{\prime} u^{\prime}\right)+x^{\prime}$ $\cdot\left(y^{\prime}+z^{\prime} v^{\prime}\right)$ while in the last case $x^{\prime} \cdot\left(y^{\prime}+z^{\prime}\right)=x^{\prime} u^{\prime} \cdot\left(z^{\prime}+y^{\prime} v^{\prime}\right)+x^{\prime} v^{\prime} \cdot\left(z^{\prime}+\right.$ $\left.y^{\prime} u^{\prime}\right)$. Hence, in every case LS $\leq$ RS completing the proof that $a \in \boldsymbol{\theta}_{3}$.

Proof of Lemma 1.1. Let $\epsilon$ be any special inclusion

$$
\rho \cdot(\sigma+\tau) \leq \phi+\chi \cdot \psi
$$

which fails in some modular lattice. For any term $\pi$, let $\pi^{0}$ denote the set of all variables occuring in $\pi$. We wish to show that $\Delta \leq \Theta_{l}[\epsilon]$. The proof is exactly the same as the proof of Lemma 2.1 in McKenzie's paper except for the last case where the sets $\rho^{0} \cap \phi^{0}, \rho^{0} \cap \psi^{0}, \tau^{0} \cap \phi^{0}, \tau^{0} \cap \chi^{0}$, and $\sigma^{0} \cap \psi^{0}$ are empty while the sets $\rho^{0} \cap \chi^{0}, \tau^{0} \cap \psi^{0}$, and $\sigma^{0} \cap \phi^{0}$ are nonempty. Suppose that, in addition, $\sigma^{0} \cap \chi^{0} \neq 0$. The assumption that various sets of variables are nonempty implies (modulo $\Lambda$ ) the inclusions $\sigma \leq \chi, \rho \leq \chi, \tau \leq \psi$, and $\sigma \leq \phi$. Hence, $M$ implies $\rho \cdot(\sigma+\tau) \leq \chi \cdot(\chi \cdot \phi+\psi)=\chi \cdot \phi+\chi \cdot \psi \leq \phi+\chi \cdot \psi$ contrary to our assumption that $\epsilon \notin M$. Hence, $\sigma^{0} \cap \chi^{0}=0$ and in this last case the following five sets are pairwise disjoint:

$$
\begin{aligned}
& \rho^{0} \cap \chi^{0}, \quad \sigma^{0} \cap \phi^{0}, \quad \tau^{0} \cap \psi^{0} \\
& \left(\rho^{0}-\chi^{0}\right) \cup\left(\tau^{0}-\psi^{0}\right) \cup\left(\sigma^{0}-\phi^{0}\right), \\
& \left(\phi^{0}-\sigma^{0}\right) \cup\left(\psi^{0}-\tau^{0}\right) \cup\left(\chi^{0}-\rho^{0}\right) .
\end{aligned}
$$


Choose three distinct variables $v_{0}, v_{1}, v_{2}$ not occuring in $\epsilon$. Replacing the variables in the five sets listed in (1), respectively, by $v_{0}, v_{1}, v_{2}, v_{0}+v_{1}+v_{2}$, $v_{0} \cdot v_{1} \cdot v_{2}$ and all remaining variables by $v_{0}$, we see that $v_{0} \cdot\left(v_{1}+v_{2}\right) \leq v_{1}+v_{0} \cdot v_{2}$ belongs to $\Theta_{l}[\epsilon]$. Since this inclusion fails in both $M_{3}$ and $N_{5}$, it implies the distributive law; thus, $\Lambda \subseteq \Theta_{l}[\epsilon]$ as desired.

Before proceeding with the proof of Lemma 1.2 we need to derive several consequences of $\alpha$, namely, $\alpha_{1}-\alpha_{4}$.

$$
\begin{aligned}
& a_{1} \cdot x \cdot(y+u+v)=x \cdot(y+u)+x \cdot(y+v)+x \cdot(u+v), \\
& a_{2} \cdot x y+z u=(x+z u) \cdot(y+z u) \cdot(z+x y) \cdot(u+x y), \\
& a_{3}\left(=a^{d}\right) \cdot x+y \cdot(z+u v)=(x+y \cdot(z+u)) \cdot(x+y \cdot(z+v)) \cdot(x+z+u v) .
\end{aligned}
$$
$(x+u+z \cdot(y+v)) \cdot(x+v+z \cdot(y+u))$,

$\alpha_{4}\left(=\alpha_{2}^{d}\right) \cdot(x+y) \cdot(z+u)=x \cdot(z+u)+y \cdot(z+u)+z \cdot(x+y)+u \cdot(x+y)$.

For the remainder of this section we let $\Theta=\Theta_{1}[\alpha]$. We first observe that the modular law belongs to $\Theta$ since

$$
x \cdot(z+y x) \sim_{\Lambda} x x \cdot(z+(y x) \cdot x) \leq_{\theta} x \cdot(y x+z \cdot(x+x)) \leq_{\Lambda} y x+z x .
$$

Actually, the modular law is equivalent (modulo $\Lambda$ ) to the inclusion $\mathrm{RS}_{\alpha} \leq \mathrm{LS}_{\alpha}$. Substituting $u+v$ for $z$ in a gives $\alpha_{1} \in \Theta$. Obviously $\mathrm{LS}_{a_{2}} \leq_{\boldsymbol{\Lambda}} \mathrm{RS}_{a_{2}}$. Now,

$$
\begin{aligned}
\mathrm{RS}_{a_{2}} & \sim_{\mathrm{M}}[(y+z u) \cdot z+x y] \cdot[(x+z u) \cdot u+x y], \\
& \sim_{\mathrm{M}}(x y+z u+y z) \cdot(x y+z u+u x), \\
& \sim_{\mathrm{M}} \mathrm{LS}_{a_{2}}+u x \cdot(x y+z u+y z),
\end{aligned}
$$

and

$$
u x \cdot(x y+z u+y z) \sim_{a_{1}} u x \cdot(x y+z u)+u x \cdot(x y+y z)+u x \cdot(z u+y z)
$$

where each of these three terms are obviously $\leq_{\Lambda}$ LS $_{a_{2}}$. Thus $a_{2} \in \Theta$. By duality $\alpha_{4} \in \Theta$ once we have shown $\alpha_{3} \in \Theta$.

It is easily seen that $\mathrm{LS}_{a_{3}} \leq_{M} \mathrm{RS}_{a_{3}}$; to illustrate we check the fourth factor on the right side: $\operatorname{LS}_{a_{3}} \leq_{\Lambda} x+(y+u v) \cdot(z+u v) \sim_{M} x+u v+z \cdot(y+u v) \leq_{\Lambda} x+$ $u+z(y+v)$. It now remains to show $\mathrm{RS}_{a_{3}} \leq_{\theta} \mathrm{LS}_{a_{3}}$. Let $\gamma$ be the product of the last four terms on the right side of $\alpha_{3}$. Then

$$
\mathrm{RS}_{a_{3}} \sim_{\boldsymbol{\theta}} \gamma \cdot(x+y z)+\gamma \cdot(x+y u)+\gamma y \cdot(z+u)+\gamma z \cdot(y+x u)+\gamma u \cdot(y+x z) .
$$

Clearly $\gamma \cdot(x+y z) \leq \mathrm{LS}_{a_{3}}$; we consider each of the remaining terms separately.

Case 1.

$$
\begin{aligned}
y u \cdot(y+x z) & \sim \sim_{\Lambda} u \cdot(y+x z) \cdot(x+y(z+v)) \cdot(x+z+u v) \cdot(x+v+z \cdot(y+u)) \\
& \leq{ }_{\theta} L_{a_{3}}+\delta_{1}+\delta_{2}+\delta_{3}+\delta_{4}
\end{aligned}
$$


where

$$
\begin{aligned}
& \delta_{1}=u \cdot(y+x z) \cdot(x+y v) \cdot(x+z+u v), \\
& \delta_{2}=u y \cdot(z+v) \cdot(x+z+u v) \cdot(x+v+z \cdot(y+u)), \\
& \delta_{3}=u \cdot(y+x z) \cdot z \cdot(y+x v) \cdot(x+v+z(y+u)) \leq_{M} \mathrm{LS}_{\alpha_{3}}, \\
& \delta_{4}=u \cdot(y+x z) \cdot v \leq_{\theta} x+y \cdot(z+u v)=\mathrm{LS}_{a_{3}} \cdot
\end{aligned}
$$

Now, $\delta_{1} \sim_{M} u \cdot(y v+x \cdot(x y+x z)) \cdot(x+z+u v) \leq_{\theta} \operatorname{LS}_{a_{3}}+\delta_{11}+\delta_{12}$ where $\delta_{11}=$ $u \cdot(y v+x y) \cdot(x+z+u v)$ and $\delta_{12}=u \cdot(y v+x z) \cdot(x+z+u v)$. It is easily seen that each $\delta_{1 i} \leq_{\theta} \mathrm{LS}_{a_{3}}$ by applying $\alpha_{1}$ to $\delta_{1 i}$ and then $\mathrm{M}$ and $\alpha$ to each summand not obviously $\leq \cdot \mathrm{LS}_{a_{3}}$. Hence, $\delta_{1} \leq_{\theta} \mathrm{LS}_{a_{3}}$. Also,

$$
\begin{aligned}
\delta_{2} & \sim{ }_{M} u y \cdot((z+u v)+x \cdot(z+v)) \cdot(x+v+z \cdot(y+u)) \\
& \leq_{\theta} \operatorname{LS}_{a_{3}}+y u \cdot(x v+z+u v)(x+v+z \cdot(y+u)) \\
& \sim{ }_{M} \operatorname{LS}_{a_{3}}+y u \cdot(z+v(x+u v))(x+v+z \cdot(y+u)) \\
& \leq{ }_{\theta} \operatorname{LS}_{a_{3}}+y u \cdot(z+v x) \cdot(x+v+z \cdot(y+u)) \\
& \sim_{M} \operatorname{LS}_{a_{3}}+y u \cdot(z \cdot(y+u)+(v+x)(z+v x)) \\
& \leq_{\theta} \operatorname{LS}_{a_{3}}+y u(z \cdot(y+u)+v x)+y u \cdot(v+x)(z+v x) .
\end{aligned}
$$

Each of these two summands are easily seen to be $\leq_{\theta} \mathrm{LS}_{\alpha_{3}}$ by applying $\alpha$ and $\mathrm{M}$ where appropriate. Thus, $\delta_{2} \leq_{\theta} \mathrm{LS}_{a_{3}}$ and hence $\gamma u(y+x z)^{3} \leq_{\theta} \mathrm{LS}_{a_{3}}$.

Case 2.

$$
\begin{aligned}
\gamma y \cdot(z+u) \sim_{M} y \cdot(z+u v+x \cdot(z+u)) \cdot(x+y \cdot(z+v)) \\
\cdot(x+u+z \cdot(y+v))(x+v+z(y+u)) \\
\leq \operatorname{LS}_{a_{3}}+\delta_{1}+\delta_{2}
\end{aligned}
$$

where

$$
\delta_{1}=y \cdot(z+u v+x u) \cdot(x+y(z+v)) \cdot(x+u+z \cdot(y+v))(x+v+z \cdot(y+u))
$$

and

$$
\delta_{2}=y u(x+z) \cdot(x+y(z+v))(x+v+z \cdot(y+u)) \leq_{\Lambda} y u \cdot(y+x z) \leq_{\boldsymbol{\theta}} \operatorname{LS}_{a_{3}} \cdot
$$

Now,

$$
\begin{aligned}
\delta_{1} \sim_{M} y \cdot(z+u \cdot(x+u v))(x+y \cdot(z+v)) \\
\\
\quad \cdot(x+u+z \cdot(y+v))(x+v+z \cdot(y+u)) \\
\leq \leq_{\theta} \operatorname{LS}_{a_{3}}+\delta_{11}+\delta_{12}
\end{aligned}
$$


where

$$
\delta_{11}=y \cdot(z+u x) \cdot(x+y \cdot(z+v)) \cdot(x+u+z \cdot(y+v)) \cdot(x+v+z \cdot(y+u))
$$

and

$$
\delta_{12}=y u \cdot(x+u v) \cdot(x+y \cdot(z+v)) \leq_{\Lambda} \gamma u \cdot(y+x z) \leq_{\theta} \operatorname{LS}_{a_{3}} \cdot
$$

Now,

$$
\delta_{11} \leq_{\theta} \mathrm{LS}_{a_{3}}+\delta_{111}+\delta_{112}+\delta_{113}
$$

where

$$
\begin{aligned}
& \delta_{111}=y \cdot(z+u x) \cdot(x+y v)(x+u+z \cdot(y+v)), \\
& \delta_{112}=y(z+u x)(z+v)(x+u+z(y+v)) \cdot(x+v+z(y+u)), \\
& \delta_{113}=y v \cdot(z+u x)(x+u+z \cdot(y+v)) \leq_{\Lambda} \delta_{111} .
\end{aligned}
$$

Now,

$$
\begin{aligned}
\delta_{111} & \sim{ }_{M} y \cdot(u x+z \cdot(x+y v)) \cdot(x+u+z \cdot(y+v)) \\
& \leq{ }_{\theta} \operatorname{LS}_{a_{3}}+y v \cdot(z+u x) \cdot(x+u+z \cdot(y+v))
\end{aligned}
$$

where the last term $\leq_{\Lambda} \gamma v \cdot(y+x z) \leq_{\theta} L_{\alpha_{3}}$ by Case 1 with $u$ and $v$ permuted. A similar argument shows $\delta_{112} \leq_{\theta} \mathrm{LS}_{a_{3}}$. Hence, $\gamma y \cdot(z+u) \leq_{\theta} \mathrm{LS}_{a_{3}}$.

\section{Case 3.}

$$
\begin{aligned}
\gamma z \cdot(y+x u) & \sim_{\Lambda} z \cdot(y+x u) \cdot(x+y \cdot(z+v)) \cdot(x+u+z \cdot(y+v)) \\
& \leq \operatorname{LS}_{a_{3}}+\delta_{1}+\delta_{2}+\delta_{3}
\end{aligned}
$$

where $\delta_{1}=z \cdot(y+x u)(x+y v) \cdot(x+u+z \cdot(y+v)), \delta_{2}=z \cdot(y+x u) \cdot(y+x v)$, and $\delta_{3}=z \cdot(y+x u) \cdot v \cdot(y+z x) \cdot(x+u+z \cdot(y+v)) \leq_{M} \operatorname{LS}_{a_{3}} \cdot$ Now,

$$
\begin{aligned}
\delta_{1} & \sim_{\mathrm{M}} z \cdot(y v+x \cdot(y+x u)) \cdot(x+u+z \cdot(y+v)) \\
& \leq \mathrm{LS}_{a_{3}}+z \cdot(y v+x u) \cdot(x+u+z \cdot(y+v)) \\
& \sim_{\mathrm{M}} \mathrm{LS}_{a_{3}}+z \cdot(x u+y v \cdot(x+u+z \cdot(y+v)) \\
& \leq_{\theta} \mathrm{LS}_{a_{3}}+\delta_{11}+\delta_{12}+\delta_{13}
\end{aligned}
$$

where $\delta_{11}=z \cdot(x u+y v \cdot(x+u)) \leq_{\theta} L_{a_{3}}+z u \cdot(y v+x u) \leq_{M} L_{a_{3}}, \delta_{12}=$ $z \cdot(x+u)(y v+x u z \cdot(y+v)) \leq_{\mathrm{M}} \mathrm{LS}_{a_{3}}$, and $\delta_{13}=z \cdot(y+v) \cdot(y v+x u) \sim_{\mathrm{M}}$ $z \cdot(y v+x u \cdot(y+v)) \leq_{\theta} \mathrm{LS}_{a_{3}}+z \cdot(y v+x v u) \leq_{\boldsymbol{\theta}} \mathrm{LS}_{a_{3}}$ since $z \cdot(y v+x u v) \leq_{\boldsymbol{\theta}} x+$ $y v(z+u v)$. Hence, $\delta_{1} \leq_{\theta} \mathrm{LS}_{a_{3}}$. Now,

$$
\delta_{2} \sim_{M} z \cdot(y+x u \cdot(y+x v)) \leq_{\theta} L_{a_{3}}+z \cdot(y+x u v) \leq_{\theta} L_{a_{3}}
$$


as with $\delta_{13}$. Hence, $\gamma z \cdot(y+x u) \leq_{\theta}$ LS $_{a_{3}}$.

Case 4.

$$
\begin{aligned}
\gamma \cdot(x+y u) & \sim_{\Lambda}(x+y u) \cdot(x+y \cdot(z+v)) \\
& \cdot(x+z+u v) \cdot(x+v+z \cdot(y+u)) \\
& \leq{ }_{\theta} \mathrm{LS}_{a_{3}}+\delta_{1}+\delta_{2}+\delta_{3}+\delta_{4}
\end{aligned}
$$

where $\delta_{1}=(x+y u) \cdot(x+y v) \cdot(x+z+u v), \delta_{2}=y \cdot(x+y u)(z+v) \cdot(x+z+$ $u v) \cdot(x+v+z \cdot(y+u)), \delta_{3}=z \cdot(x+y u)(y+x v) \cdot(x+v+z \cdot(y+u)), \delta_{4}=$ $v \cdot(x+y u)(y+x z) \cdot(x+z+u v)$. Now,

$\delta_{1} \sim_{\mathrm{M}}(x+y u) \cdot(x+y v \cdot(x+z+u v)) \sim_{\mathrm{M}} x+y v(x+y u) \cdot(x+z+u v) \leq_{\Lambda} x+\delta_{4}$

and $\delta_{i} \leq_{\theta} \mathrm{LS}_{a_{3}}$ (for $i=2,3,4$ ) follows from Cases 2, 3, and 1 respectively with variables $u$ and $v$ interchanged. Hence, $\gamma \cdot(x+y u) \leq_{\theta} L_{a_{3}}$ completing the proof that $a_{3} \in \boldsymbol{\Theta}_{l}[a]$.

Proof of Lemma 1.2. We only prove (i); (ii) follows by duality. The proof is only a slight modification of McKenzie's proof of Lemma 2.2. Let $F$ (resp. G), with or without subscripts, always denote a finite, nonempty set of ST1's (resp. ST1's and terms of the type $\zeta+\sigma$, where $\zeta$ and $\sigma$ are products of variables).

First, note that given $F$, we can find a $G$ with $\Sigma G \leq_{M} \Sigma F$ and $x \cdot \Sigma F \sim_{\theta}$ $\Sigma\{x \cdot \phi: \phi \in G\}$. Indeed, by $\alpha_{1}$,

$$
x \cdot \sum_{F \sim_{\theta}} \sum\left\{x \cdot\left(\phi_{0}+\phi_{1}\right): \phi_{0}, \phi_{1} \in F\right\}
$$

and each $\phi_{0}+\phi_{1} \leq_{M} \Sigma F$. By several applications of $\alpha$ : if $\phi_{K}=\zeta_{K} \cdot\left(\sigma_{\kappa 0}+\kappa_{\kappa 1}\right)$, then (where $\kappa, \lambda, \delta$ range over $\{0,1\}$ )

$$
\begin{aligned}
x \cdot\left(\phi_{0}+\phi_{1}\right) \sim_{\theta} & \sum_{\kappa, \lambda} x \cdot\left(\zeta_{0} \cdot \sigma_{0 \kappa}+\zeta_{1} \cdot \sigma_{1}\right)+\sum_{\kappa} x \cdot \zeta_{\kappa} \cdot\left(\sigma_{\kappa 0}+\sigma_{\kappa 1}\right) \\
& +x \cdot \zeta_{0} \cdot \sigma_{10} \cdot \sigma_{11} \cdot\left(\sigma_{00}+\sigma_{01}\right) \\
& +\sum_{\kappa, \lambda, \delta} x \cdot \sigma_{\kappa \lambda} \cdot\left(\zeta_{\kappa}+\zeta_{1-\kappa} \cdot \sigma_{\kappa 1-\lambda} \cdot \sigma_{1-\kappa \delta}\right) \\
& +\sum_{\kappa, \lambda} x \cdot \sigma_{1 \kappa} \sigma_{0 \lambda} \cdot\left(\sigma_{11-\kappa} \cdot \zeta_{0}+\zeta_{1} \cdot \sigma_{01-\lambda}\right) .
\end{aligned}
$$

$G$ is a subset of $F$ together with the above five types of $\phi$ 's associated with pairs $\phi_{0}, \phi_{1}$ in $F$. We need only check that for each of the types of elements $\phi$ described, $\phi \leq_{M} \Sigma F$. For $\phi$ a member of $F$ or one of the first three types above it is obvious that $\phi \leq_{M} \Sigma F$. Suppose $\phi=\sigma_{1 \kappa} \cdot \sigma_{0 \lambda} \cdot\left(\sigma_{11-\kappa} \cdot \zeta_{0}+\zeta_{1} \cdot \sigma_{01-\lambda}\right)$, i.e., is of the fifth type. Then

$$
\phi \leq_{\Lambda}\left(\sigma_{00}+\sigma_{01}\right) \cdot\left(\sigma_{11-\kappa} \cdot \zeta_{0}+\zeta_{1} \cdot\left(\sigma_{00}+\sigma_{01}\right)\right) \leq_{M} \sigma_{11-\kappa} \cdot \zeta_{0} \cdot\left(\sigma_{00}+\sigma_{01}\right)+\zeta_{1} \cdot
$$

Therefore, 


$$
\begin{aligned}
\phi & \leq_{M} \sigma_{1 \kappa} \cdot\left(\zeta_{1}+\phi_{0} \cdot \sigma_{11-\kappa}\right) \leq_{\Lambda}\left(\sigma_{10}+\sigma_{11}\right) \cdot\left(\zeta_{1}+\phi_{0} \cdot\left(\sigma_{10}+\sigma_{11}\right)\right) \\
& \leq_{M} \phi_{1}+\phi_{0} \leq \dot{\sum}_{F} .
\end{aligned}
$$

Thus, $\phi \leq_{M} \Sigma F$. For $\phi=\sigma_{K \lambda} \cdot\left(\zeta_{K}+\zeta_{1-K} \cdot \sigma_{\kappa 1-\lambda} \cdot \sigma_{1-K \delta}\right)$ of the fourth type a similar argument shows

$$
\phi \leq_{M} \zeta_{K} \cdot\left(\sigma_{\kappa 0}+\sigma_{\kappa 1}\right)+\zeta_{1-\kappa} \cdot \sigma_{1-\kappa \delta} \cdot\left(\sigma_{\kappa 0}+\sigma_{\kappa 1}\right) \leq \phi_{K}+\phi_{1-\kappa} \leq \sum F .
$$

This completes our preliminary remarks. (i) is proved by induction on the formation of terms. The only nontrivial part of the argument is the passage over products: assume that $\sigma_{\kappa} \sim_{\theta} \Sigma F_{\kappa}$ and $\Sigma F_{\kappa} \leq_{M} \sigma_{K}$ (for $\left.\kappa=0,1\right)$ and consider $\sigma_{0} \cdot \sigma_{1}$. By the above we have sets $G_{0}, G_{1}$ such that

$$
\sigma_{0} \cdot \sigma_{1} \sim_{\theta} \sum\left\{\sigma_{0} \cdot \phi_{1}: \phi_{1} \in G_{1}\right\} \sim_{\theta} \sum\left\{\phi_{0} \cdot \phi_{1}: \phi_{1} \in G_{1}, \phi_{0} \in G_{0}\right\}
$$

where each term $\phi_{0} \cdot \phi_{1} \leq_{M} \sigma_{0} \cdot \sigma_{1}$. Thus, it only remains to consider the simple terms $\phi_{0} \cdot \phi_{1}$. Suppose $\phi_{\kappa}=\zeta_{K} \cdot\left(\sigma_{\kappa 0}+\sigma_{\kappa 1}\right)$ for $\kappa=0,1$. We apply $\alpha_{4}, \alpha_{1}$ and then $\alpha$ repeatedly as in the construction of $G$ above to see that $\phi_{0} \cdot \phi_{1}$ is equivalent modulo $\Theta$ to sums of ST1's of the type that occur.in (2). Repeating our previous argument shows that, for each such ST1 $\nu, \nu \leq_{M} \phi_{0} \cdot \phi_{1}$ and hence $\nu \leq_{M} \sigma_{0} \cdot \sigma_{1}$ as desired. This completes the proof of Lemma 1.2.

The following corollary which is an easy consequence of Lemmas 1.1 and 1.2 was suggested to us by Professor McKenzie.

Corollary 1.5. An inclusion $\sigma \leq \tau$ is valid in $M_{3}$ iff every special inclusion $\nu \leq \phi$, for which $\nu \leq_{M} \sigma$ and $\tau \leq_{M} \phi$, is provable in $M$.

2. We will now show that Schützenberger's identity $\beta$ does not characterize $0 N_{5}$. Since $\beta$ is rather complicated it is useful to observe that it is equivalent to the following two identities:

$$
\begin{aligned}
& \beta_{1} \cdot x \cdot(y+z)=x \cdot(x y+z)+x \cdot(y+x z), \\
& \beta_{2} \cdot x \cdot(w+y \cdot(u+v))=x y \cdot(u+v)+x \cdot(y u+w)+x \cdot(y v+w) .
\end{aligned}
$$

The identity $\beta_{1}$ is just McKenzie's $\eta_{3} ; \beta_{2}$ is the dual of $\eta_{7}$.

Suppose $Q_{3}$ is the lattice given in Figure 1 . Observe that $Q_{3}$ is subdirectly irreducible, self-dual and has a nontrivial automorphism $\phi$. Our results are based on the following lemma.

Lemma 2.1. $\beta_{2}$ bolds in $Q_{3}$.

As a consequence of this lemma, $Q_{3}$ gives a counterexample to Schützenberger's claim.

Theorem 2.2. The identity $\beta$ bolds in $Q_{3}$ but $Q_{3}$ is not a member of $\vartheta_{N_{5}}$.

Proof. That $Q_{3} \notin \mathcal{O} N_{5}$ is mentioned in McKenzie's paper. For a short direct proof we need only observe that McKenzie's identity $\eta_{1}, x \cdot(y+u) \cdot(y+v) \leq$ $x \cdot(y+u v)+x u+x v$, fails in $Q_{3}$ when $f, e, g$ and $b$ are assigned to $x, y, u$, and $v$ 
respectively. The fact that $\beta \in \Theta Q_{3}$ follows from Lemma 2.1 , the self-duality of $Q_{3}$ and the remark that $\beta_{1} \in \boldsymbol{\Theta}_{l}\left[\beta_{2}^{d}\right]$. To see that $\beta_{1}$ is a consequence of $\Lambda$ and $\beta_{2}^{d}, x+w(y+u v)=(x+y+u v) \cdot(x+w \cdot(y+u)) \cdot(x+w \cdot(y+v))$, we first observe

$$
w \cdot(x+y) \leq_{\beta_{2}^{d}} x+w \cdot(y+x w)
$$

This follows from $\beta_{2}^{d}$ by setting $u=x$ and $v=w$. Thus,

$$
x \cdot(x y+z)+x \cdot(y+x z) \sim_{\beta_{2}^{d}}[x(x y+z)+y] \cdot x \cdot(y+z) \sim_{\beta_{2}^{d}} x \cdot(y+z)
$$

where the last equality holds by (1).

Proof of Lemma 2.1. Suppose the elements $x^{\prime}, y^{\prime}, w^{\prime}, u^{\prime}$, and $v^{\prime}$ in $Q_{3}$ are substituted for the variables $x, y, w, u$ and $v$ in $\beta_{2}$ respectively. The left (similarly, the right) side of $\beta_{2}$ is assigned the value LS (similarly RS). It is obvious that RS $\leq$ LS; thus, it suffices to show LS is always equal to a sum of the values on the right. This is obviously true if either $w^{\prime} \geq y^{\prime}$ or $w^{\prime} \geq u^{\prime}+v^{\prime}$ or $u^{\prime}$ and $v^{\prime}$ are comparable. We assume

$$
w^{\prime} \unrhd y^{\prime}, w^{\prime} \unrhd u^{\prime}+v^{\prime} \text {, and } u^{\prime} \text { and } v^{\prime} \text { are incomparable. }
$$

In view of the automorphism $\phi$ and the fact that $L S \leq R S$ whenever $w^{\prime} \in\{0,1\}$, it is enough to show $\mathrm{LS} \leq \mathrm{RS}$ whenever $w^{\prime} \in\{a, f, g, e, c\}$. If $w^{\prime}$ is incomparable with $u^{\prime}+v^{\prime}$, then either $w^{\prime}=a$ and $u^{\prime}+v^{\prime}=b$ or $w^{\prime}=g$ and $u^{\prime}+v^{\prime} \in\{e, b\}$. In the first case either $u^{\prime}=b$ or $v^{\prime}=b$ so LS $=x^{\prime}=x^{\prime} \cdot\left(y^{\prime} u^{\prime}+w^{\prime}\right)+x^{\prime} \cdot\left(y^{\prime} v^{\prime}+w^{\prime}\right)$; in the second, if $u^{\prime}+v^{\prime}, y^{\prime} \in\{1, b, b\}$ then LS $=x^{\prime}=x^{\prime} \cdot\left(y^{\prime} u^{\prime}+w^{\prime}\right)+$ $x^{\prime} \cdot\left(y^{\prime} y^{\prime}+w^{\prime}\right)$ and if either $u^{\prime}+v^{\prime}=e$ or $y^{\prime} \in\{\alpha, f, e, d\}$, LS $=x^{\prime} a=$ $x^{\prime} \cdot\left(y^{\prime} u^{\prime}+w^{\prime}\right)+x^{\prime} \cdot\left(y^{\prime} v^{\prime}+w^{\prime}\right)$. Hence, we may assume

$$
w^{\prime}<u^{\prime}+v^{\prime} \text {. }
$$

If $w^{\prime}<y^{\prime}$, then $y^{\prime} \cdot\left(u^{\prime}+v^{\prime}\right) \geq w^{\prime}$ so LS $=x^{\prime} y^{\prime} \cdot\left(u^{\prime}+v^{\prime}\right)$; hence, we may also assume

$$
w^{\prime} \text { and } y^{\prime} \text { are incomparable. }
$$

From (2), (3), (4) it remains to consider four cases

(6.1) $w^{\prime}=a, y^{\prime} \in\{b, b\}, u^{\prime}+v^{\prime}=1$,

(6.2) $w^{\prime} \in\{f, e\}, y^{\prime}=g, u^{\prime}+v^{\prime} \in\{1, a, b\}$,

(6.3) $w^{\prime}=g, y^{\prime} \in\{f, e, b, b, d\}, u^{\prime}+v^{\prime} \in\{1, a\}$,

(6.4) $w^{\prime}=c, y^{\prime} \in\{d, b\}, u^{\prime}+v^{\prime} \in\{1, a, b, e\}$.

To illustrate, we consider (6. 4). If $u^{\prime}+v^{\prime} \in\{a, e\}$, either $u^{\prime}$ or $v^{\prime}$ belong to $\{d, e, f\}$; thus, $\mathrm{LS}=x^{\prime} \cdot e=x^{\prime} \cdot\left(y^{\prime} u^{\prime}+w^{\prime}\right)+x^{\prime} \cdot\left(y^{\prime} v^{\prime}+w^{\prime}\right)$. On the other hand, if $u^{\prime}+v^{\prime} \in\{1, b\}$, either $u^{\prime}$ or $v^{\prime}$ belongs to $\{b, b\}$; so either $y^{\prime} \cdot u^{\prime}=y^{\prime}$ or $y^{\prime} \cdot v^{\prime}=$ $y^{\prime}$. Thus LS $=x^{\prime} \cdot\left(w^{\prime}+y^{\prime}\right)=x^{\prime} \cdot\left(y^{\prime} u^{\prime}+w^{\prime}\right)+x^{\prime} \cdot\left(y^{\prime} v^{\prime}+w^{\prime}\right)$ as desired. The 
other cases are, likewise, easily checked. We conclude that $\mathrm{LS} \leq \mathrm{RS}$, and hence $\beta_{2}$, always holds in $Q_{3}$.

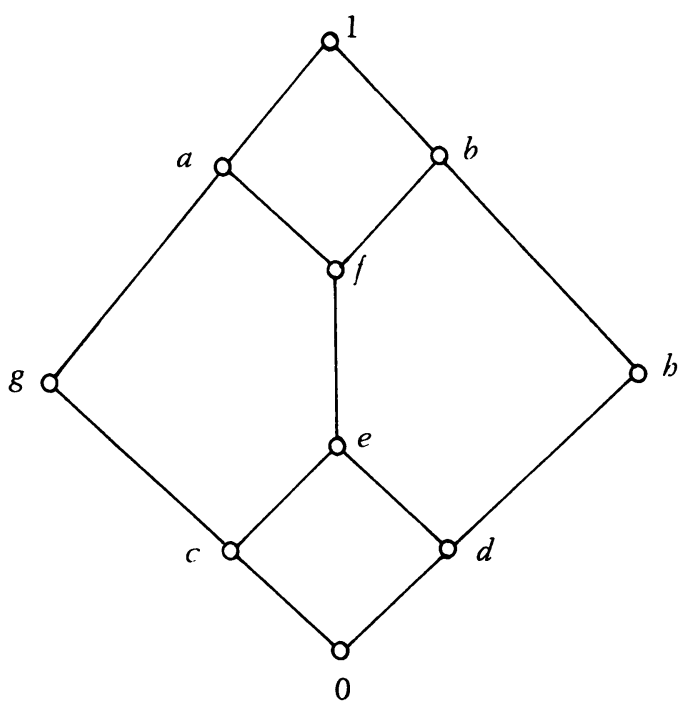

Figure 1

BIBLIOGRAPHY

1. B. Jónsson, Equational classes of lattices, Math. Scand. 22 (1968), 187-196. MR $40 \# 66$.

2. R. McKenzie, Equational bases and nonmodular lattices varieties, Trans. Amer. Math. Soc. 174 (1972), 1-43.

3. M. Schützenberger, Sur certains axiomes de la théorie des structures, C. R. Acad. Sci. Paris 221 (1945), 218-220. MR 7, 235.

DEPARTMENT OF MATHEMATICS, VANDERBILT UNIVERSITY, NASHVILLE, TENNESSEE 37203

Current address: (D. X. Hong) Department of Mathematics, University of Saigon, Saigon, Republic of Viet-Nam 